\title{
A low complexity PAPR reduction scheme based on radix-II IFFT
}

\begin{abstract}
Due to no feedback process and simplicity in searching algorithm, conventional selected mapping (CSLM) is an efficient crest factor reduction (CFR) technique in orthogonal frequency division multiplexing (OFDM) systems. However high number of inverse fast Fourier transform (IFFT) block is required to achieve the desired PAPR reduction performance. In this paper a PAPR reduction method based on $\mathrm{N}$ point radix-2 IFFT is proposed in which the number of IFFTs is reduced to one. The gist of the proposed method is based on storing a part of calculations and using them for the next searching operation results in elimination of the redundant calculations. Simulation results show at least $46.8 \%$ complexity reduction compared to CSLM by comparable PAPR performance.
\end{abstract}

Keyword: PAPR reduction; Radix-2 inverse fast Fourier transform; OFDM; IFFT 